\title{
The Inverse Problem of Variational Calculus with Non-holonomic Constraints
}

\author{
Pedro Gonçalves Henriques \\ Departamento de Matemática, Instituto Superior Técnico \\ Av. Rovisco Pais, 1, 1049-001 Lisboa, Portugal \\ E-mail address: phenr@math.ist.utl.pt \\ Dedicated to Luís Magalhães and Carlos Rocha for the occasion of their 60th birthdays.
}

\begin{abstract}
We will discuss some new results for the inverse problem of Variational Calculus. We will consider problems with functionals given by action forms of order greater than one and subject to non-holonomic constraints.
\end{abstract}

\section{Introduction}

Griffiths (see [16]) presented a new approach to variational problems in the context of exterior differential systems, and proposed mixed endpoint conditions for problems with non-holonomic constraints to obtain stationary solutions. With these non-holonomic constraints it is generally not possible to have variations of integral manifolds subject to fixed endpoint conditions. These mixed endpoint conditions will make the integral over the boundary of the first variation vanish. In [26] we generalized Griffiths's framework to variational problems given by multiple integrals, and established mixed boundary conditions for variational problems with nonholonomic constraints. The study of Variational Calculus for functionals defined by multiple integrals was developed by Caratheodory [1929], WeilDe Donder [1936], Lepage [1936-1942]. Other authors like Dedecker [19531977], Liesen [1967], R. Hermann [1966], H. Goldschmidt and S. Sternberg [1973], Ouzilou [1972], D. Krupka [1970-1975], I. M. Anderson [1980], P. L. Garcia and A. Pérez-Rendón [1969-1978], C. Günther [1987], Edelen [1961] and Rund [1966] contributed with their work to this field.

Key words: Inverse problem of Variational Calculus. Non-holonomic constraints.

Research supported by the Center for Mathematical Analysis, Geometry, and Dynamical Systems. 
In 1887, Helmholtz presented the inverse problem of Variational Calculus in the following way: Given $P_{i}=P_{i}\left(x, u^{j}, u_{x}^{j}, u_{x x}^{j}\right)$, is there a Lagrangian $L\left(x, u^{j}, u_{x}^{j}\right)$ such that $E_{i}(L)=\partial L / \partial u^{i}-D_{x} \partial L / \partial u_{x}^{i}=P_{i}$, where $D_{x}=$ $\partial / \partial x+u_{x}^{i} \partial / \partial u^{i}+u_{x x}^{i} \partial / \partial u_{x}^{i}$ ? Necessary conditions were found for $P_{i}$ to be a Euler-Lagrange system (see (3.1) (3.2) and (3.3)). These conditions were proved to be locally sufficient.

I. M. Anderson [1992], [1980], P. J. Olver [1986], F. Takens [1979], W. M. Tulczyjew [1980] and A. M. Vinagradov [1964] generalized Helmholtz's conditions both for higher order systems of partial differential equations and for multiple integrals.

In the present text we describe new results for the the inverse problem of Variational Calculus for multiple integrals in the context of exterior differential systems. We deal with non-holonomic constraints in the setting of the mixed boundary conditions defined in [26]. This work is a follow up of [29] and [30]. In section I and II we present a short review of the latter work. In section III we discuss the inverse problem of Variational Calculus, and conclude in section IV with a study of the generalized Lagrange problem with non-holonomic constraints.

1.1. Integral manifolds and valued differential systems. Let us consider a manifold $\mathrm{X}$ and two subbundles of the cotangent bundle $T^{*} X$, satisfying:

i) $I^{*} \subset T^{*} X$,

ii) $L^{*} \subset T^{*} X$ with $I^{*} \subset L^{*} \subset T^{*} X$,

with the $\operatorname{rank}\left(L^{*} / I^{*}\right)=n$ (a natural number).

We define an integral manifold of $\left(I^{*}, L^{*}\right)$ as an oriented connected compact $n$-dimensional smooth manifold $N$ together with a smooth mapping

$$
f: N \rightarrow X
$$

satisfying:

for all $x \in N$.

$$
I_{f(x)}^{*} \stackrel{\perp}{=} L_{f(x)}^{*} \stackrel{\perp}{ }+f_{*}(T N)
$$

$N$ may admit a piecewise smooth boundary $\partial N$.

$V\left(I^{*}, L^{*}\right)$ is the collection of integral manifolds $f$ of $\left(I^{*}, L^{*}\right)$.

Let $\varphi$ be an n-form on $X$. A valued differential system of $\left(I^{*}, L^{*}\right)$ is a triple $\left(I^{*}, L^{*}, \varphi\right)$.

We define the functional $\phi$ associated with $\left(I^{*}, L^{*}, \varphi\right)$ in $V\left(I^{*}, L^{*}\right)$ by:

$$
\phi: V\left(I^{*}, L^{*}\right) \rightarrow R,
$$




$$
f \rightarrow \phi[f]=\int f^{*} \varphi
$$

1.2. Local embeddability. Let us define a Pfaffian differential system which is locally embeddable in $J^{1}\left(R^{n}, R^{m}\right)$. The differenial system for the Lagrange problem will be defined later [26]. Let $d\left(C^{\infty}\left(X, L^{*}\right)\right) \subset$ $C^{\infty}\left(X, L^{*} \wedge T^{*} X\right)$ and $d^{\prime}=\operatorname{dim} X, s=\operatorname{rank} I^{*}$. We denote $d\left(C^{\infty}\left(X, L^{*}\right)\right.$ as the set of images obtained by the exterior derivative of $C^{\infty}\left(X, L^{*}\right)$. We can set for every $p \in X$ a chart coordinate system $\left\{u^{1}, \ldots, u^{s+n}, v^{1}, \ldots, v^{d^{\prime}-s-n}\right\}$ so that:

i)

$$
\left.L^{*}=\operatorname{span}\left\{d u^{\alpha} \mid 1 \leq \alpha \leq s+n\right\}\right)
$$

ii)

$$
L^{* \perp}=\operatorname{span}\left\{\frac{\partial}{\partial v} \mid 1 \leq i \leq d^{\prime}-s-n\right\}
$$

for an open subset $U$ of $X$ with $p \in U$, using the Frobenius theorem. Let $\delta$ be the map $I^{*} \wedge \Omega \rightarrow \Lambda^{n+1}\left(T^{*} U\right) / I_{u}^{*} \wedge\left(\Lambda^{n}(T * U)\right)$ induced by:

$$
d: C^{\infty}\left(U, I^{*} \wedge \Omega\right) \rightarrow C^{\infty}\left(U, \Lambda^{n+1}\left(T^{*} U\right)\right)
$$

in $I^{*} \wedge \Omega$.

Definition 1.1. A Pfaffian differential system $\left(I^{*}, L^{*}\right)$ with $d\left(C^{\infty}(X, L)\right) \subset$ $C^{\infty}\left(X, L^{*} \wedge T^{*} X\right)$ is locally embeddable if for every $p \in X$ there exists an open neighborhood $U$ of $p$ and local coframes $C F=\left\{\theta_{1}, \ldots, \theta_{s}\right\}$ for $I_{U}^{*}$ and $C F^{\prime}=\left\{\theta_{1}, \ldots, \theta_{s}, d u^{\prime s+1}, d u^{\prime s+n}\right\}$ for $L_{U}^{*}$, satisfying:

(i) $\left.\delta\left(I_{U}^{*} \wedge \Omega\right) \subset T^{*} U \wedge \Lambda^{n}\left(L_{U}^{*}\right) / T^{*} U \wedge I_{U}^{*} \wedge \Lambda^{n-1}\left(L^{*}\right)\right)$,

(ii) $\operatorname{Ker} \delta$ is a constant rank subbundle of $I^{*} \wedge \Omega$,

where $\Omega=\operatorname{span}\left\{d u " s+1 \wedge \ldots \wedge \widehat{d u^{\prime s+j}} \wedge \ldots \wedge d u^{n s+n}\right\}$,

$\widehat{d u " s+j}$ - means deletion of $s+j$ factor (for $n=1, \widehat{d u " s+1}=1$ ).

If $I^{*}$ has no Cauchy characteristics, the structure equations are locally:

$$
\begin{aligned}
& d \theta^{\alpha} \equiv \pi_{j}^{\alpha} \wedge d u^{" s+j}+A_{\alpha^{\prime} \beta}^{\alpha j^{\prime}} \pi_{j^{\prime}}^{\alpha^{\prime}} \wedge \theta^{\beta}+B_{\beta j}^{\alpha} \theta^{\beta} \wedge d u^{\prime s+j} \bmod I \wedge I \\
& 1 \leq \alpha, \alpha^{\prime}, \beta \leq s, 1 \leq j, j^{\prime}, j^{\prime \prime} \leq n, I=C^{\infty}\left(X, I^{*}\right) .
\end{aligned}
$$


1.3. The Cartan system. We will now define the Cartan system whose solutions, when projected in $X$, will be candidates for extremum of $\phi$ for appropriate boundary conditions.

We begin by assuming that $\left(I^{*}, L^{*}, \varphi\right)$ is a valued differential system on $X$, and that $W$ is the total space of $I^{*}$. Let us consider $\chi$ the canonical form on $T^{*} X$, and $i$ the inclusion map $W \stackrel{i}{\hookrightarrow} T^{*} X$.

We assume that the $n$-form $\omega$ is locally given by:

$$
\omega=\omega^{1} \wedge \ldots \wedge \omega^{n}
$$

inducing a nonzero section of $\Lambda^{n}\left(L^{*} / I^{*}\right)$.

$$
\omega_{i}=(-1)^{i-1} \omega^{1} \wedge \ldots \wedge \widehat{\omega^{i}} \ldots \wedge \omega^{n} .
$$

$W^{n}$ is the $n$-Cartesian power of $W$. We define $Z$ as a subset of $W^{n}$ by $Z=\left\{z \in W^{n}: \pi^{\prime}(z) \in X\right\}$, where $\pi^{\prime}$ is the projection $\pi^{\prime}(z): W^{n} \rightarrow X$, and $\Delta X^{n}$ is the diagonal submanifold of $X^{n}$.

$Z$ is a vector subbundle over $X$ and $\operatorname{dim} Z=d+s n$. Let $\psi$ be

$$
\psi=\pi^{*} \varphi+\left(\pi^{j} o i^{\prime}\right)^{*}\left[i^{*}(\chi)\right] \wedge \pi^{*} \omega_{j} .
$$

$\pi^{j}$ is the natural projection into the $j^{\text {th }}$ component $\pi^{j}: W^{n} \rightarrow W$, i' is the inclusion map $Z \rightarrow W^{n}, \pi$ is the natural projection $\pi: Z \rightarrow X$ and

$$
\Psi=d \psi .
$$

Locally, $\left(\pi^{j} o i^{\prime}\right)^{*}\left[i^{*}(\chi)\right] \wedge \pi^{*} \omega_{j}=\lambda_{j}^{i} \theta_{i}{ }^{j}$ with $\theta_{i}{ }^{j}=\theta^{j} \wedge \omega_{j}$.

Definition 1.2. The Cartan system $C(\Psi)$ is the ideal generated by the set of $n$-forms

$$
\left.\{v\lrcorner \Psi \quad \text { where } v \in C^{\infty}(Z, T Z)\right\} .
$$

Integral manifolds of $(C(\Psi), \omega)$ are oriented connected compact n-dimensional smooth manifolds $N$ (possibly with a piecewise smooth boundary $\partial N$ ) together with a smooth mapping $f: N \rightarrow X$, satisfying:

$$
f^{*} \theta=0 \quad \text { for every } \quad \theta \in C(\Psi)
$$

and

$$
f^{*}(\omega) \neq 0 .
$$

We can now express the first variation of $\phi$ by:

$$
\left.\left.\delta \phi=\int_{f(N)} v\right\lrcorner d \psi+d(v\lrcorner \psi\right) .
$$


1.4. The momentum space. Let us assume that we have on $Z$ :

(i) a closed $(n+1)$-form $\Psi$ with the associated Cartan system $C(\Psi)$,

(ii) $\pi^{\prime *} \omega$ is the pull-back to $Z$ of $\omega$, which is the $n$-form inducing a nonzero section on $\wedge^{n}\left(L^{*} / I^{*}\right)$.

Definition 1.3. Let $\left(C(\Psi), \pi^{* *} \omega\right)_{n}$ be the ideal generated by $\left(C(\Psi), \pi^{\prime *} \omega\right)$ in $C^{\infty}\left(Z, \wedge^{n} T^{*} Z\right)$. We say that $\left[z_{0}, E_{0}^{p}\right]$, with $z_{0} \in Z$ and $E_{0}^{p}$ a $p$-dimensional subspace of the tangent space $T_{z_{0}}$, is a p-dimensional integral element of $\left(C(\Psi), \pi^{* *} \omega\right)_{n}$ if

(i) $<E_{0}^{p}, \alpha>=0$ for all $\left(C(\Psi), \pi^{\prime *} \omega\right)_{n}$,

(ii) $\left.<E_{0}^{p}\right\lrcorner \omega>\neq 0$.

$\left.V_{n}\left(C(\Psi), \pi^{*} \omega\right)\right)$, the set of integral elements $\left[z_{0}, E_{0}^{p}\right]$, is a subset of $G_{n}(Z)$. Let $\pi$ " be the projection $G_{n}(Z) \rightarrow Z$. Let us assume that:

$$
\begin{gathered}
\left.\left.Z_{1}=\pi "\left(V_{n}\left(C(\Psi), \pi^{*} \omega\right)\right), V_{n}^{\prime}\left(C(\Psi), \pi^{*} \omega\right)\right)\right)= \\
\left\{E \in V_{n}\left(C(\Psi), \pi^{*} \omega\right): E \text { tangent to } Z_{1}\right\}, \\
\left.\left.Z_{2}=\pi^{\prime \prime}\left(V_{n}^{\prime}\left(C(\Psi), \pi^{*} \omega\right)\right), V^{\prime \prime}{ }_{n}\left(C(\Psi), \pi^{*} \omega\right)\right)\right)= \\
\left\{E \in V_{n}^{\prime}\left(C\left(\Psi, \pi^{*} \omega\right): E \text { tangent to } Z_{2}\right\} \ldots\right.
\end{gathered}
$$

are subundles of $Z$.

Definition 1.4. Let $\left(I^{*}, L^{*}, \varphi\right)$ be a locally embeddable valued differential system, and $\omega=\omega^{1} \wedge \ldots \wedge \omega^{n}$. If there exists a $k_{0} \in \mathbb{N}$, such that in the above construction $Z_{k_{0}}=Z_{k_{0}+1}=\ldots=Z_{k_{0}+n^{\prime}}\left(n^{\prime} \in N\right)$, with

(i) $Z_{k_{0}}$ a manifold of dimension $(n+1) m+n$ for $m \in \mathbb{N}$, and

(ii) $\left(C(\Psi), \pi^{*} \omega\right)_{Z_{k_{0}}}$ being a differential system in $Z_{k_{0}}$ with $r_{n}=0$ (Cartan number in Cartan-Kähler Theorem) for all $\left.V_{n-1}\left(C(\Psi), \pi^{*} \omega\right)\right)$,

then $\left(I^{*}, L^{*}, \varphi\right)$ is a non-degenerate valued differential system. We will rename $Z_{k_{0}}$ the momentum space $Y$.

For $n=1$ we follow [16] and replace condition (ii) by $\psi \wedge \Psi^{n} \neq 0$ on $Z_{k_{0}}$.

We call $\left(C(\Psi), \pi^{*} \omega\right)_{Y}$ the prolongation of $\left(C(\Psi), \pi^{*} \omega\right)$ in the momentum space. In this construction, the differential system $\left(C(\Psi), \pi^{*} \omega\right)_{Y}$ satisfies:

(i) the projection $\left(C(\Psi), \pi^{*} \omega\right) \rightarrow Y$ is surjective,

(ii) and the integral manifolds of $\left(C(\Psi), \pi^{\prime *} \omega\right)$ on $Z$ coincide with those of $\left(C(\Psi), \pi^{*} \omega\right)$ on $Y$. 
1.5. Well-posed valued differential systems. Let us assume that

(a) we have the following subbundles of $T^{*} X$

$$
\begin{array}{ccccc}
I^{*} & \subset & L^{*} & \subset & T^{*} X \\
P^{*} & & M^{*}
\end{array}
$$

(b) the locally given $\mathrm{n}$-form $\omega$ also induces a nonzero section on $\Lambda^{n}\left(M^{*} / P^{*}\right)$, and

(c) $\left.Y \subset\left(P^{*}\right)^{n}\right|_{\Delta X^{n}}$, with $Y$ being a subbundle of $\left.\left(P^{*}\right)^{n}\right|_{\Delta X^{n}}$.

Definition 1.5. $\left(I^{*}, L^{*}, \varphi, P^{*}, M^{*}\right)$ is a well-posed valued differential system if we have the following conditions fulfilled:

(i) $\left(I^{*}, L^{*}, \varphi\right)$ is a non-degenerate valued differential system (with $\operatorname{dim} Y=(n+1) m+n)$ and $\varphi=L \omega$ for a smooth function $L$ on $X$,

(ii) there exists a subbundle $P^{*}$ of $I^{*}$ of rank $m$ and a subbundle $M^{*}$ of $L^{*}$ of rank $m+n$ as in (1.15),

(iii) $\pi^{* *} M^{*}=\operatorname{span}\left\{\pi^{*} \theta \mid \theta \in C^{\infty}\left(X, M^{*}\right)\right\}$ is completely integrable on $Y$, where $\pi "=\pi \circ i$, with $i$ once more denoting the inclusion mapping $Y \rightarrow Z$ and $\pi$ the projection $Z \rightarrow X$.

$C F=\left\{\theta^{\alpha}, d u^{s+j}, \pi_{j^{\prime}}^{\alpha^{\prime}}, \pi_{j}^{\alpha^{\prime \prime}} \mid 1 \leq \alpha \leq s, 1 \leq \alpha^{\prime} \leq s_{l}, j^{\prime} \in L_{\alpha^{\prime}}, s_{l+1} \leq \alpha " \leq\right.$ $s, 1 \leq j \leq n\}$ for $T^{*} X$ with $L_{\alpha^{\prime}} \subset\{k \in N, 1 \leq k \leq n\}$ :

(i)

$$
I^{*}=\operatorname{span}\left\{\theta^{\alpha} \mid 1 \leq \alpha \leq s\right\}
$$

(ii)

$$
L^{*}=\operatorname{span}\left\{\theta^{\alpha}, d u^{s+j} \mid 1 \leq \alpha \leq s, 1 \leq j \leq n\right\},
$$

(iii) $T^{*} X=L^{*} \oplus R^{*}\left(\oplus\right.$ denotes a direct sum) with $R^{*}=\operatorname{span}\left\{\pi_{j^{\prime}}^{\alpha^{\prime}}, \pi_{j}^{\alpha^{\prime \prime}} \mid 1 \leq\right.$ (iv) $\left.\alpha^{\prime} \leq s_{l}, j^{\prime} \in L_{\alpha^{\prime}}, s_{l+1} \leq \alpha^{\prime \prime} \leq s, 1 \leq j \leq n\right\}$,

$$
d \theta_{j^{\prime \prime}}^{\alpha^{\prime}} \equiv 0 \bmod I \text {, for } j " \notin L_{\alpha^{\prime}}\left\{\theta_{j}^{\alpha^{\prime}}=\theta^{\alpha^{\prime}} \wedge \omega_{j^{\prime \prime}}\right\},
$$

(v)

$$
d \theta_{j^{\prime}}^{\alpha^{\prime}} \equiv \pi_{j^{\prime}}^{\alpha^{\prime}} \wedge \omega \bmod I, \text { for } j^{\prime} \in L_{\alpha^{\prime}},
$$

$$
d \theta_{j}^{\alpha "} \equiv \pi_{j}^{\alpha "} \wedge \omega \bmod I, \text { when } 1 \leq j \leq n,
$$

(vii) $\pi_{j^{\prime}}^{\alpha^{\prime}}, \pi_{j}^{\alpha "}$ are linearly independent $\bmod L$. 
In [29], we presented a set of boundary conditions for different types of well-posed valued differential systems. For these boundary conditions, solutions of the Cartan system are solutions of the Euler-Lagrange system. (These have null first variations.)

\section{Generalized Lagrange problem}

The framework for this Lagrange problem with or without constrains represents a set of problems that is highly relevant to the study of Calculus of Variations.

The generalized Lagrange problem is defined on $X=J^{1}\left(\mathbb{R}^{n}, \mathbb{R}^{m}\right)$ (the 1 jet manifold), with the canonical system $I^{*}$ defined on $X$ (i.e. $I^{*}=$ $\left.\operatorname{span}\left\{\theta^{\alpha}=d y^{\alpha}-y_{x^{i}}^{\alpha} d x^{i}\right\}\right)$ and $\varphi=L \omega$, with $\omega=d x^{1} \wedge \ldots \wedge d x^{n}$. We choose $x^{1}, \ldots, x^{n}$ to be coordinates for $\mathbb{R}^{n}$, and $y^{1}, \ldots, y^{m}$ coordinates for $\mathbb{R}^{m}$.

Definition 2.1. Let $f$ be a solution to the canonical differential system $I^{*}$, with the independence condition given by $L^{*}=\operatorname{span}\left\{I^{*}, d x^{1}, \ldots, d x^{n}\right\}$. A family $F\left(x, t_{1}, \ldots, t_{k}\right)$ of integral manifolds of $\left(I^{*}, L^{*}\right)$ is a $k$-parameter variation of $f k \in \mathbb{N}$ if:

(i) $F\left(x, t_{1}, \ldots, t_{k}\right)$ is smooth with $\left(t_{1}, \ldots, t_{k}\right) \in\left[0, \epsilon_{1}\right] \times \ldots \times\left[0, \epsilon_{k}\right]$, for $\epsilon_{i}>0,1 \leq i \leq k$

(ii) $F_{\left(t_{1}, \ldots, t_{k}\right)} \doteq \bar{F}\left(x, t_{1}, \ldots, t_{k}\right) \in V\left(I^{*}, L^{*}\right)$ for all $\left(t_{1}, \ldots, t_{k}\right) \in\left[0, \epsilon_{1}\right] \times$ $\ldots \times\left[0, \epsilon_{k}\right]$,

(iii) $F(x, 0)=f(x)$ for all $x \in N, N \subset \mathbb{R}$.

$F_{*}\left(\frac{\partial}{\partial t_{i}}\right)$ is an infinitesimal variation of $F$.

We will consider variations satisfying the condition $\pi^{\prime \prime}(F(x, t))=\pi "(f(x))$ for all $x \in \partial N$ and $t \in[0, \epsilon] \quad\left(\pi\right.$ " is the projection $\left.J^{1}\left(R^{n}, R^{m}\right) \rightarrow R^{n}\right)$.

Without loss of generality we can choose $v$ so that $v\lrcorner d x^{i}=0$, thus replacing a one parameter variation of $f$ by another that has the same first and second variation while satisfying:

$$
\pi "(F(x, t))_{N}=i d_{N}
$$

for all $t \in[0, \epsilon])$.

\section{Inverse problem for calculus of variations}

3.1. First example. In 1887, Helmholtz solved the following problem:

Example 1. Given $P_{\alpha}=P_{\alpha}\left(x, u^{\beta}, u_{x}^{\beta}, u_{x x}^{\beta}\right)$. Is there a Lagrangian $L\left(x, u^{\beta}, u_{x}^{\beta}\right)$ such that $E_{\alpha}(L)=\partial L / \partial u^{\alpha}-D_{x} \partial L / \partial u_{x}^{\alpha}=P_{\alpha}$, where $D_{x}=$ $\partial / \partial x+u_{x}^{\alpha} \partial / \partial u^{\alpha}+u_{x x}^{\alpha} \partial / \partial u_{x}^{\alpha}$ ? He found the necessary conditions for $P_{\alpha}$ : 


$$
\begin{aligned}
\partial P_{\alpha} / \partial u_{x x}^{\beta} & =\partial P_{\beta} / \partial u_{x x}^{\alpha} \\
\partial P_{\alpha} / \partial u_{x}^{\beta} & =\partial P_{\beta} / \partial u_{x}^{\alpha}+2 D_{x} \partial P_{\beta} / \partial u_{x x}^{\alpha} \\
\partial P_{\alpha} / \partial u^{\beta} & =\partial P_{\beta} / \partial u^{\alpha}-D_{x} \partial P_{\beta} / \partial u_{x}^{\alpha}+D_{x x} \partial P_{\beta} / \partial u_{x x}^{\alpha} .
\end{aligned}
$$

Let $E \rightarrow M$ be a fibered manifold. $J^{\infty}(E)$ (see [5] and [41]) is the infinite jet of $E$.

Let

and

$$
\begin{aligned}
\theta^{i} & =d u^{\alpha}-u_{x}^{\alpha} d x \\
\theta_{x}^{\alpha} & =d u_{x}^{\alpha}-u_{x x}^{\alpha} d x
\end{aligned}
$$

$$
\begin{gathered}
\Omega_{P}=P_{\alpha} \theta^{\alpha} \wedge d x+1 / 2\left[\partial P_{\alpha} / \partial u_{x}^{\beta}-D_{x} \partial P_{\alpha} / \partial u_{x x}^{\alpha}\right] \theta^{\beta} \wedge \theta^{\beta} \\
+1 / 2\left[\partial P_{\alpha} / \partial u_{x x}^{\alpha}+\partial P_{\beta} / \partial u_{x x}^{\alpha}\right] \theta^{\alpha} \wedge \theta_{x}^{\beta} .
\end{gathered}
$$

If $P$ satisfies the Helmholtz conditions (3.1), (3.2) and (3.3), then $d \Omega_{P}=$ 0 . If $\Omega_{P}$ is exact (equivalently, if $H^{n+1}(E) \mathrm{n}+1$, de Rham cohomology group of $E$ is trivial), then $P_{\alpha}$ is globally variational.

If $\theta_{L}=L d x+\partial L / \partial u_{x}^{\alpha} \theta^{\alpha}$, then $d \theta_{L}=\Omega_{P}$.

Volterra [51] showed that if $L=\int_{N} u^{\alpha} P_{\alpha}\left(x, t u^{\beta}, t u_{x}^{\beta}, t u_{x x}^{\beta}\right) d t$, where $N=$ $[0,1]$, then:

$$
E_{\alpha}(L)=P_{\alpha} .
$$

We obtain a global solution to the inverse problem in the case of one independent variable and $P_{\alpha}=0$ equations of second order.

In 1964, Vaingberg [50] generalized this result. However, this Lagrangian is usually of much higher order than necessary.

From [5] one can derive the following theorem:

Theorem 3.1. Let $\Delta$ be a differential operator of order $2 k$

$$
\Delta=P_{\alpha}\left(x^{i}, u^{\beta}, u_{1}^{\beta}, \ldots, u_{2 k}^{\beta}\right) \theta^{\beta} \wedge \omega .
$$

Then $\Delta$ is the Euler-Lagrange operator of a $k^{\text {th }}$-order Lagrangian $L\left(x^{i}, u^{\beta}, u_{1}^{\beta}, \ldots, u_{k}^{\beta}\right)$, if and only if $\Delta$ satifies the higher order Helmholtz conditions, and the functions

$$
P_{\alpha}(t)=P_{\alpha}\left(x^{i}, u^{\beta}, u_{1}^{\beta}, \ldots, u_{k}^{\beta}, t u_{k+1}^{\beta} \ldots, t^{k} u_{2 k}^{\beta}\right)
$$

are polynomials in $t$ of degree less or equal to $k$.

$u_{k}^{\beta}$ denote all possible $k^{t h}$ - order derivatives of $u^{\beta}, 1 \leq \alpha, \beta \leq m$ and $1 \leq i \leq n, \theta^{\beta}=d u^{\beta}-u_{x^{i}}^{\beta} d x^{i}$ and $\omega=d x^{1} \wedge \ldots \wedge d x^{n}$. 
3.2. Variational Bicomplex. Let us recall now a very important tool for a globalization of the inverse problem [5], [41].

Definition 3.1. A p-form $\omega$ on $J^{\infty}(E)$ is said to be of type $(r, s)$, where $r+s=p$, if at each point $x$ of $J^{\infty}(E)$

$$
\omega\left(X_{1}, X_{2}, \ldots, X_{p}\right)=0,
$$

whenever either

(i) more than $s$ of the vectors $X_{1}, X_{2}, \ldots, X_{p}$ are $\pi_{M}^{\infty}$ vertical, or

(ii) more than $r$ of the vectors $X_{1}, X_{2}, \ldots, X_{p}$ annihilate all contact one forms.

Note: $\Omega^{r, s}$ denotes the space of type $(r, s)$ forms on $J^{\infty}(E)$.

(i) $\pi: E \rightarrow M$ is a fiber bundle.

(ii) There exists a set of differential equations on sections of $E$.

$$
\begin{gathered}
d=d_{H}+d_{V} \\
d_{H}: \Omega^{r, s}\left(J^{\infty}(E)\right) \rightarrow \Omega^{r+1, s}\left(J^{\infty}(E)\right), \\
d_{V}: \Omega^{r, s}\left(J^{\infty}(E)\right) \rightarrow \Omega^{r, s+1}\left(J^{\infty}(E)\right), \\
d_{H}^{2}=0, d_{H} d_{V}=-d_{V} d_{H}, d_{V}^{2}=0 .
\end{gathered}
$$

In local coordinates

$$
\begin{gathered}
d_{H} f=\left[\partial f / \partial x^{i}+u_{x^{i}}^{\alpha} \partial f / \partial u^{\alpha}+u_{x^{i} x^{j}}^{\alpha} \partial f / \partial u_{x^{j}}^{\alpha}+\ldots\right] d x^{x^{i}}, \\
d_{V} f=\partial f / \partial u^{\alpha} \theta^{\alpha}+\partial f / \partial u_{x^{i}}^{\alpha} \theta_{x^{i}}^{\alpha}+\ldots
\end{gathered}
$$

$I$ is locally given by:

$$
\begin{gathered}
I: \Omega^{r, s}\left(J^{\infty}(E)\right) \rightarrow \Omega^{r, s}\left(J^{\infty}(E)\right), \\
\left.I(\omega)=\frac{1}{s} \theta^{\alpha} \wedge\left[\left(\partial / \partial u^{\alpha}\right\lrcorner \omega\right)-D_{x^{i}}\left(\left(\partial / \partial u_{x^{i}}^{\alpha}\right\lrcorner \omega\right)+D_{x^{i} x^{j}}\left(\left(\partial / \partial u_{x^{i} x^{j}}^{\alpha}\right\lrcorner \omega\right)-\ldots\right]
\end{gathered}
$$

Definition 3.2. The sequences of spaces

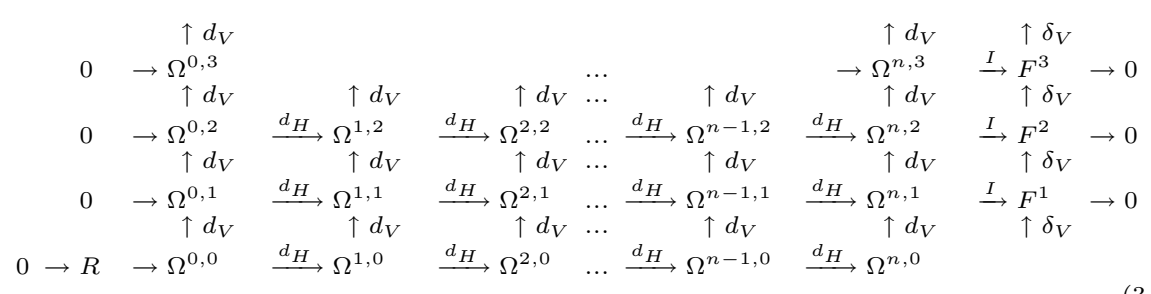

is the Variational Bicomplex. 
Therefore, we have:

$0 \rightarrow R \rightarrow \Omega^{0,0} \stackrel{d_{H}}{\longrightarrow} \Omega^{1,0} \stackrel{d_{H}}{\longrightarrow} \Omega^{2,0} \ldots \stackrel{d_{H}}{\longrightarrow} \Omega^{n-1,0} \stackrel{d_{H}}{\longrightarrow} \Omega^{n, 0} \stackrel{E}{\longrightarrow} F^{1} \stackrel{\delta_{H}}{\longrightarrow} F^{2} \stackrel{\delta_{H}}{\longrightarrow} F^{3} \ldots$

\subsection{Two other examples.}

Example 2. $\operatorname{Let} T=T\left(x^{i}, u, u_{x^{i}}, \ldots, u_{x^{i_{1}} x^{i_{2}}}\right)$ be a second order operator $1 \leq$ $i_{1}, i_{2} \leq n$. We assume that $T$ is a smooth function. Let $L=L\left(x^{i}, u, u_{x^{i}}\right)$ be a first-order operator, with $L$ being a smooth function. $E[L]=\partial L / \partial u-$ $D_{x^{i}} \partial L / \partial u_{x^{i}}$, where $D_{x^{i}}=\partial / \partial x^{i}+u_{x^{i}} \partial / \partial u+u_{x^{i}, x^{i_{1}}} \partial / \partial u_{x^{i_{1}}}+\ldots$

Let $v$ be a lift to the momentum space of an infinitesimal variation $F_{*}(\partial / \partial t)$ of $f=\pi o g$, where $g$ is a solution of $\left(C(\Psi), \pi^{*} \omega\right)$. The Liederivative of $\psi=\pi^{*} \varphi+\left(\pi^{\beta} o i^{\prime}\right)^{*}\left[i^{*}(\chi)\right] \wedge \pi^{*} \omega_{\beta}$ along $v$ is:

$$
\begin{aligned}
& \left.v\lrcorner d \psi+d(v\lrcorner \psi)=E[L](u) v^{1} \pi^{*}\left(d x^{1} \wedge \ldots \wedge d x^{n}\right)\right) \\
& +d\left(\partial L / \partial u_{x^{i}} v^{1} \pi^{*}\left((-1)^{i-1} d x^{1} \wedge \ldots \wedge \widehat{d x^{i}} \ldots \wedge d x^{n}\right) .\right.
\end{aligned}
$$

If we identify $e_{i}$ with $\pi^{*}\left((-1)^{i-1} d x^{1} \wedge \ldots \wedge \widehat{d x^{i}} \ldots \wedge d x^{n}\right)$ at each point of the integral manifold of $\left(C(\Psi), \pi^{*} \omega\right)$, we can write:

where

$$
\begin{gathered}
d\left(\partial L / \partial u_{x^{i}} v^{1} \pi^{*}\left((-1)^{i-1} d x^{1} \wedge \ldots \wedge \widehat{d x^{i}} \ldots \wedge d x^{n}\right)\right) \\
=\operatorname{Div} V[u] \pi^{*}\left(d x^{1} \wedge \ldots \wedge d x^{n}\right),
\end{gathered}
$$

$$
V[u]=\partial L / \partial u_{x^{i}} v^{i} e_{i}
$$

We have

$$
E[L](u)=0 \quad \text { if } \quad L[u]=\operatorname{Div} W[u]
$$

and

$$
H(T)=0 \quad \text { if } \quad T[u] \quad \text { is Euler-Lagrange. }
$$

Helmholtz equations are:

$$
\partial T / \partial u_{x^{i}}=D_{x^{i}} \partial T / \partial u_{x^{i} x^{i}}+1 / 2 D_{x^{i_{1}}} \partial T / \partial u_{x^{i} x^{i_{1}}} .
$$

There exists a sequence of spaces

$$
V(u) \stackrel{\text { Div }}{\longrightarrow} F(u) \stackrel{E}{\longrightarrow} F(u) \stackrel{H}{\longrightarrow} V(u)
$$

that is a cochain complex, the Euler-Lagrange complex, where $F[u]$ is the set of smooth functions $F\left(x^{i}, u, u_{x^{i}}, \ldots, u_{x^{i_{1}}} x^{i_{2}}\right), V[u]$ is the set of vector fields defined in $\mathbb{R}^{n}$ with $F[u]$ coeficients. This is a particular case of (3.19). 
This complex is exact and thus the inverse problem is solved in this second example.

Example 3. Let $T_{\alpha}\left(x^{i}, u^{\beta}, u_{x^{i}}^{\beta}, \ldots, u_{x^{i_{1} x^{i_{2}}}}^{\beta}\right)$ be second-order operators $1 \leq$ $i_{1}, i_{2} \leq n$ and $1 \leq \beta, \alpha \leq m$. We assume that $T_{\alpha}$ are smooth functions. Let $L=L\left(x^{i}, u^{\beta}, u_{x^{i}}^{\beta}\right)$ be a first-order operator, with $L$ being a smooth function. $E_{\alpha}[L]=\partial L / \partial u^{\alpha}-D_{x^{i}} \partial L / \partial u_{x^{i}}^{\alpha}$ where $D_{x^{i}}=\partial / \partial x^{i}+u_{x^{i}}^{\alpha} \partial / \partial u^{\alpha}+$ $u_{x^{i}, x^{i_{1}}}^{\alpha} \partial / \partial u_{x^{i_{1}}}^{\alpha}+\ldots$

Helmholtz equations are:

$$
\begin{aligned}
\partial T_{\alpha} / \partial u_{x^{i} x^{i_{1}}}^{\beta} & =\partial T_{\beta} / \partial u_{x^{i} x^{i_{1}}}^{\alpha} \\
\partial T_{\alpha} / \partial u_{x^{i}}^{\beta} & =\partial T_{\beta} / \partial u_{x^{i}}^{\alpha}+2 D_{x^{i_{1}}} \partial T_{\alpha} / \partial u_{x^{i} x^{i_{1}}}^{\alpha} \\
\partial T_{\alpha} / \partial u^{\beta} & =\partial T_{\beta} / \partial u^{\alpha}-D_{x^{i}} \partial T_{\beta} / \partial u_{x^{i}}^{\alpha}+D_{x^{i} x_{1} i_{1}} \partial T_{\beta} / \partial u_{x^{i} x^{i_{1}}}^{\alpha}
\end{aligned}
$$

\section{G.L. problem with non-holonomic constraints}

4.1. G.L. problem with non-holonomic constraints. Let us recall from [26] the generalized Lagrange problem with non-holonomic constraints for $n>1, m>1$.

Let us assume $g^{\rho}\left(x^{i}, u^{\alpha}, u_{x^{j}}^{\alpha}\right)=0$, with $\operatorname{rank}\left[\partial g^{\rho} / \partial u_{x^{j}}^{\alpha}\right]=m n-l$, $g^{\rho}\left(x^{i}, u^{\alpha}, u_{x^{j}}^{\alpha}\right)$ are smooth functions, with $1 \leq i, j \leq n, 1 \leq \alpha \leq m$, $1 \leq \rho \leq m n-l$ and $l \geq 0$. $\left(I^{*}, L^{*}, \varphi, I^{*}, L^{*}\right)$ is a well-posed valued differential system, where: $I^{*}=\operatorname{span}\left\{\theta^{\alpha}\right\}$, and $L^{*}=\operatorname{span}\left\{\theta^{\alpha}, d x^{i} \mid 1 \leq i \leq n\right\}$

and

$$
\theta^{\alpha}=\left\{d u^{\alpha}-u_{x^{i}}^{\alpha} d x^{i} \mid 1 \leq i \leq n\right\}
$$

$$
\left.\theta_{j}^{\alpha}=\theta^{\alpha} \wedge \omega_{j} \mid 1 \leq i \leq n\right\} .
$$

In this setting we have:

$$
\begin{gathered}
d \theta_{i}^{\mu}=-d u_{x^{i}}^{\mu} \wedge \omega, \\
d \theta_{i_{\sigma}}^{\sigma}=-d u_{x^{i \sigma}}^{\sigma} \wedge \omega \quad \text { with } i_{\sigma} \in L_{\sigma} \subset\{1, \ldots, \mathrm{n}\} \\
d \theta_{j_{\sigma}}^{\sigma}=+A_{j_{\sigma} \mu}^{i \sigma} d u_{x^{i}}^{\mu} \wedge \omega+A_{j_{\sigma} \sigma^{\prime}}^{i_{\sigma^{\prime}}} d u_{x^{\sigma^{\prime}}}^{\sigma^{\prime}} \wedge \omega \\
+B_{j_{\sigma} \alpha}^{\sigma} \theta^{\alpha} \wedge \omega \bmod I \wedge I \operatorname{with} j_{\sigma} \notin L_{\sigma} . \\
L_{x^{i}}^{\mu}=\left(\partial / \partial u_{x^{i}}^{\mu}-A_{j_{\sigma} \mu}^{i \sigma} \partial / \partial u_{x^{j \sigma}}^{\sigma}\right) L, \\
L_{x^{\sigma^{\prime} \sigma^{\prime}}}^{\sigma^{\prime}}=\left(\partial / \partial u_{x^{i^{\prime}}}^{\sigma^{\prime}}-A_{j_{\sigma} \sigma^{\prime}}^{i_{\sigma^{\prime}} \sigma} \partial / \partial u_{x^{j \sigma}}^{\sigma}\right) L
\end{gathered}
$$




$$
\begin{aligned}
L_{x^{i^{\prime}} x^{i}}^{\nu \mu} & =\left(\partial / \partial u_{x^{i}}^{\mu}-A_{j_{\sigma} \mu}^{i \sigma} \partial / \partial u_{x^{j \sigma}}^{\sigma}\right) L_{x^{i^{\prime}}}^{\nu}, \\
L_{x^{i} x^{i} \sigma^{\prime}}^{\mu \sigma^{\prime}} & =\left(\partial / \partial u_{x^{i} \sigma^{\prime}}^{\sigma^{\prime}}-A_{j_{\sigma} \sigma^{\prime}}^{i_{\sigma^{\prime}} \sigma} \partial / \partial u_{x^{j \sigma}}^{\sigma}\right) L_{x^{i}}^{\mu}, \\
L_{x^{i} \sigma^{\prime} x^{i}}^{\sigma^{\prime}} & =\left(\partial / \partial u_{x^{i}}^{\mu}-A_{j_{\sigma} \mu}^{i \sigma} \partial / \partial u_{x^{j \sigma}}^{\sigma}\right) L_{x^{i} \sigma^{\prime}}^{\sigma^{\prime}}, \\
L_{x^{\sigma^{\prime} \sigma^{\prime} x^{i} \sigma^{\prime \prime}}}^{\sigma^{\prime}} & =\left(\partial / \partial u_{x^{i} \sigma^{\prime \prime}}^{\sigma^{\prime \prime}}-A_{j_{\sigma} \sigma^{\star}}^{i \sigma} \partial / \partial u_{x^{j \sigma}}^{\sigma}\right) L_{x^{i} \sigma^{\prime}}^{\sigma^{\prime}},
\end{aligned}
$$

with $1 \leq \sigma, \sigma^{\prime} \sigma^{\prime \prime} \leq m-m_{l}$ and $m-m_{l}+1 \leq \mu, \nu \leq m$.

We have $\sum_{\sigma=1}^{m_{l}} n_{\sigma}=l$, where $n_{\sigma}=n-\# L_{\sigma}$.

$$
\begin{gathered}
\Psi \equiv\left(L_{x^{i}}^{\mu}-\lambda_{x^{i}}^{\mu}-\lambda_{j_{\sigma}}^{\sigma} A_{j_{\sigma} \mu}^{i \sigma}\right) \pi^{*}\left(d u_{x^{i}}^{\mu} \wedge \omega\right) \\
+\left(L_{x^{i} \sigma^{\prime}}^{\sigma^{\prime}}-\lambda_{x^{i} \sigma^{\prime}}^{\sigma^{\prime}}-\lambda_{j_{\sigma}}^{\sigma} A_{j_{\sigma} \sigma^{\prime}}^{i_{\sigma^{\prime}} \sigma}\right) \pi^{*}\left(d u_{x^{i \sigma}}^{\sigma} \wedge \omega\right) \\
+d \lambda_{i}^{\mu} \wedge \pi^{*}\left(\theta_{i}^{\mu}\right)+d \lambda_{i}^{\sigma} \wedge \pi^{*}\left(\theta_{i}^{\sigma}\right) \\
+\left(L_{u^{\alpha}}-\lambda_{j_{\sigma}}^{\sigma} B_{j_{\sigma} \alpha}^{\sigma}+L_{u_{x^{\sigma^{\prime} \sigma^{\prime}}}} B_{j_{\sigma} \alpha}^{\sigma}\right) \pi^{*}\left(\theta^{\alpha} \wedge \omega\right) \bmod I \wedge I,
\end{gathered}
$$

The Cartan system is:

$$
\begin{aligned}
& \pi^{*} \theta_{i}^{\alpha} \quad(1 \leq \alpha \leq m \quad \text { and } \quad 1 \leq i \leq n), \\
& \left(L_{x^{i}}^{\mu}-\lambda_{x^{i}}^{\mu}-\lambda_{j_{\sigma}}^{\sigma} A_{j_{\sigma} \mu}^{i \sigma}\right) \pi^{*} \omega \quad\left(m-m_{l}+1 \leq \mu \leq m\right), \\
& \left(L_{x^{i^{\prime}}}^{\sigma^{\prime}}-\lambda_{x^{i} \sigma^{\prime}}^{\sigma^{\prime}}-\lambda_{j_{\sigma}}^{\sigma} A_{j_{\sigma} \sigma^{\prime}}^{i_{\sigma^{\prime}} \sigma}\right) \pi^{*} \omega \\
& \left(1 \leq \sigma, \sigma^{\prime}, \sigma^{\text {" }} \leq m_{\sigma} \text { with } i_{\sigma^{\prime}} \in L_{\sigma} \text { and } j_{\sigma} \notin L_{\sigma}\right) \\
& \left(-d \lambda_{x^{i}}^{\mu} \wedge \pi^{*} \omega_{i}\right)+\left(L_{u^{\mu}}-\lambda_{j_{\sigma}}^{\sigma} B_{j_{\sigma} \mu}^{\sigma}+L_{u_{x^{\sigma^{\prime}}{ }^{\prime}}} B_{j_{\sigma} \mu}^{\sigma}\right) \wedge \pi^{*} \omega \\
& \left(-d \lambda_{x^{j} \sigma^{\prime \prime}}^{\sigma^{\prime \prime}} \wedge \pi^{*} \omega_{j_{\sigma^{\prime \prime}}}-d \lambda_{x^{j_{\sigma} \prime}}^{\sigma^{\prime \prime}} \wedge \pi^{*} \omega_{i_{\sigma^{\prime \prime}}}\right) \\
& +\left(L_{u^{\sigma^{\prime \prime}}}-\lambda_{j_{\sigma}}^{\sigma} B_{j_{\sigma} \sigma^{\text {" }}}^{\sigma}+L_{u_{x^{j^{\prime} \sigma^{\prime}}}} B_{j_{\sigma} \sigma^{\prime \prime}}^{\sigma}\right) \wedge \pi^{*} \omega .
\end{aligned}
$$

Let us assume $g^{\rho} / \partial u_{x^{i}}^{\mu}=0$ for all $m_{l}+1 \leq \mu \leq m$ and $g^{\rho} / \partial u_{x_{\sigma}^{i}}^{\sigma}=0$ for all $i_{\sigma} \in L_{\sigma}$ and $1 \leq \sigma \leq m_{l}$. Then the Euler-Lagrange equations are:

$$
E^{\mu}(L)=\partial L / \partial u^{\mu}-D_{x^{i}} \partial L / \partial u_{x^{i}}^{\mu}+\partial L / \partial u_{x^{i} \sigma^{\prime}}^{\sigma^{\prime}} B_{j_{\sigma} \mu}^{\sigma}+\lambda_{j_{\sigma}}^{\sigma} B_{j_{\sigma} \mu}^{\sigma}
$$




$$
E^{\sigma}(L)=\partial L / \partial u^{\sigma}-D_{x^{i \sigma}} \partial L / \partial u_{x^{i} \sigma}^{\sigma}+\partial L / \partial u_{x^{i^{\prime}}}^{\sigma^{\prime}} B_{j_{\sigma}^{\prime} \sigma}^{\sigma^{\prime}}+\lambda_{j_{\sigma}^{\prime}}^{\sigma^{\prime}} B_{j_{\sigma}^{\prime} \sigma}^{\sigma^{\prime}}-\lambda_{j_{\sigma} x^{i} \sigma}^{\sigma}
$$

Proposition 4.1. Let $\left(I^{*}, L^{*}\right)$ be a locally embeddable differential system defined on $X=\left.J^{1}\left(\mathbb{R}^{n}, \mathbb{R}^{m}\right)\right|_{g^{\rho}\left(x^{i}, u^{\alpha}, u_{x^{j}}^{\alpha}\right)=0}, \operatorname{rank}\left[\partial g^{\rho} / \partial u_{x^{j}}^{\alpha}\right]=m n-l$, $g^{\rho}\left(x^{i}, u^{\alpha}, u_{x^{j}}^{\alpha}\right)$ are smooth functions $(1 \leq i, j \leq n, 1 \leq \alpha \leq m, 1 \leq \rho \leq$ $m n-l, l \geq 0)$, and $g^{\rho} / \partial u_{x^{i}}^{\mu}=0$ for all $m_{l}+1 \leq \mu \leq m$ and $g^{\rho} / \partial u_{x_{\sigma}^{i}}^{\sigma}=0$ and for all $i_{\sigma} \in L_{\sigma}$ and $1 \leq \sigma \leq m_{l}$, where $I^{*}=\operatorname{span}\left\{\theta^{\alpha}\right\}, L^{*}=\operatorname{span}$ $\left\{\theta^{\alpha}, d x^{i} \mid 1 \leq i \leq n\right\}$

$$
\theta^{\alpha}=d u^{\alpha}-u_{x^{j}}^{\alpha} d x^{j} \quad 1 \leq j \leq n
$$

Let

and

$$
Q_{\mu}\left(x^{i}, u^{\mu}, u_{x^{i}}^{\mu}, u_{x^{i} x^{j}}^{\mu}, u^{\sigma}, u_{x^{i \sigma}}^{\sigma}, u_{x^{i \sigma} x^{i^{\prime}}}^{\sigma}, \lambda_{j_{\sigma}}^{\sigma}, \lambda_{j_{\sigma} x^{i \sigma}}^{\sigma}\right)
$$

$$
Q_{\sigma}\left(x^{i}, u^{\mu}, u_{x^{i}}^{\mu}, u_{x^{i} x^{j}}^{\mu}, u^{\sigma}, u_{x^{i} \sigma}^{\sigma}, u_{x^{i \sigma} x^{i^{\prime}}}^{\sigma}, \lambda_{j_{\sigma}}^{\sigma}, \lambda_{j_{\sigma} x^{i \sigma}}^{\sigma}\right),
$$

with $m_{l}+1 \leq \mu \leq m, 1 \leq \sigma \leq m_{l}$ and $1 \leq i \leq n$ and $\left(i_{\sigma}, i_{\sigma^{\prime}}\right) \in\left(L_{\sigma}\right)^{2}$, with

$$
Q_{\alpha}\left(x^{i}, u^{\sigma}, u_{x^{i}}^{\mu}, t u_{x^{i} x^{j}}^{\mu}, u^{\sigma}, u_{x^{i \sigma}}^{\sigma}, t u_{x^{i \sigma} x^{i_{\sigma}}}^{\sigma}, \lambda_{j_{\sigma}}^{\sigma}, \lambda_{j_{\sigma} x^{i_{\sigma}}}^{\sigma}\right)
$$

$1 \leq \alpha \leq m$ being polynomials in $t$ of degree less or equal to 1 , and

$$
\begin{gathered}
P_{\sigma}=Q_{\sigma}+\lambda_{j_{\sigma}^{\prime}}^{\sigma^{\prime}} B_{j_{\sigma^{\prime}} \sigma}^{\sigma^{\prime}}-\lambda_{j_{\sigma} x^{i} \sigma}^{\sigma}, \\
P_{\mu}=Q_{\mu}+\lambda_{j_{\sigma}}^{\sigma} B_{j_{\sigma} \mu}^{\sigma} .
\end{gathered}
$$

Furthermore, if we assume that $P_{\alpha}$ satisfy Helmholtz conditions and do not depend on $\lambda_{j_{\sigma}}^{\sigma}$ and $\lambda_{j_{\sigma} x^{i} \sigma}^{\sigma}$ coordinates, then $Q_{\alpha}$ are locally Euler-Lagrange operators for a Lagrangian $L\left(x^{i}, u^{\mu}, u_{x^{i}}^{\mu}, u^{\sigma}, u_{x^{i}}^{\sigma}\right)$.

Proof: In this case the Helmholtz conditions are:

$$
\begin{aligned}
\partial P_{\alpha} / \partial u_{x^{i} x^{i_{1}}}^{\beta} & =\partial P_{\beta} / \partial u_{x^{i} x^{i_{1}}}^{\alpha}, \\
\partial P_{\alpha} / \partial u_{x^{i}}^{\beta} & =\partial P_{\beta} / \partial u_{x^{i}}^{\alpha}+2 D_{x^{i_{1}}} \partial P_{\alpha} / \partial u_{x^{i} x^{i_{1}}}^{\alpha}, \\
\partial P_{\alpha} / \partial u^{\beta} & =\partial P_{\beta} / \partial u^{\alpha}-D_{x^{i}} \partial P_{\beta} / \partial u_{x}^{\alpha}+D_{x^{i} x^{i_{1}}} \partial P_{\beta} / \partial u_{x^{i} x^{i_{1}}}^{\alpha} .
\end{aligned}
$$

From Theorem 3.1 we know that a function $F\left(x^{i}, u^{\mu}, u_{x^{i}}^{\mu}, u^{\sigma}, u_{x^{i \sigma} \sigma}^{\sigma}\right)$ can be found such that $E_{\alpha}[F]=P_{\alpha}$.

In addition, if in the domain of $P_{\alpha}$ the sequence of spaces is exact

$$
\Omega^{n, 0} \stackrel{E}{\rightarrow} F^{1} \stackrel{H}{\longrightarrow} 0,
$$

then we have a global solution for the inverse problem. 
Example 4. Let $X=\left.J^{1}\left(\mathbb{R}^{n}, \mathbb{R}^{m}\right)\right|_{g^{\rho}\left(x^{i}, u^{\alpha}, u_{x^{i}}^{\alpha}\right)=0}, \operatorname{rank}\left[\partial g^{\rho} / \partial u_{x^{j}}^{\alpha}\right]=m n-l$, $g^{\rho}\left(x^{i}, u^{\alpha}, u_{x^{i}}^{\alpha}\right)$ are smooth functions $(1 \leq i, j \leq n, 1 \leq \alpha \leq m, 1 \leq \rho \leq n-$ $l, l \geq 0)$. Furthermore, let us assume that $g^{\rho} / \partial u_{x^{i}}^{\mu}=0$ for all $m_{l}+1 \leq$ $\mu \leq m$ and $g^{\rho} / \partial u_{x_{\sigma}^{i}}^{\sigma}=0$ for all $i_{\sigma} \in L_{\sigma}, 1 \leq \sigma \leq m_{l} . I^{*}=\operatorname{span}\left\{\theta^{\alpha}\right\}$, $L^{*}=\operatorname{span}\left\{\theta^{\alpha}, d x^{i} \mid 1 \leq i \leq n\right\}$.

$$
\begin{aligned}
& Q_{\sigma}\left(x^{i}, u^{\mu}, u_{x^{i}}^{\mu}, u_{x^{i}}^{\mu} x^{j}, u^{\sigma}, u_{x^{i}}^{\sigma}, u_{x^{i} \sigma x^{i^{\prime} \sigma}}^{\sigma}, \lambda_{j_{\sigma}}^{\sigma}, \lambda_{j_{\sigma} x^{i} \sigma}^{\sigma}\right)= \\
& 2 u_{x^{j^{\prime}}}^{\sigma^{\prime}} B_{j_{\sigma^{\prime}} \alpha}^{\sigma}+\sum_{i_{\sigma}} 2 u_{x^{i \sigma} x^{i \sigma}}^{\sigma}-\lambda_{j_{\sigma}}^{\sigma} B_{j_{\sigma} \sigma}^{\sigma}-\lambda_{j_{\sigma} x^{i \sigma}}^{\sigma}, \\
& Q_{\mu}\left(x^{i}, u^{\mu}, u_{x^{i}}^{\mu}, u_{x^{i}}^{\mu} x^{i}, u^{\sigma}, u_{x^{i_{\sigma}}}^{\sigma}, u_{x^{i_{\sigma}} x^{i_{\sigma}^{\prime}}}^{\sigma}, \lambda_{j_{\sigma}}^{\sigma}, \lambda_{j_{\sigma} x^{i_{\sigma}}}^{\sigma}\right)= \\
& 2 u_{x^{j \sigma}}^{\sigma}\left(x^{i}, u\right) B_{j_{\sigma} \alpha}^{\mu}+\sum_{i} 2 u_{x^{i} x^{i}}^{\mu}-\lambda_{j_{\sigma}}^{\sigma} B_{j_{\sigma} \sigma}^{\sigma} .
\end{aligned}
$$

$Q_{\sigma}-\lambda_{j_{\sigma}}^{\sigma} B_{j_{\sigma}^{\prime} \sigma}^{\sigma^{\prime}}-\lambda_{j_{\sigma}^{\prime} x^{i_{\sigma}}}^{\sigma^{\prime}}$ and $Q_{\mu}-\lambda_{j_{\sigma}}^{\sigma} B_{j_{\sigma} \mu}^{\mu}$ satisfy Helmhotz equations and

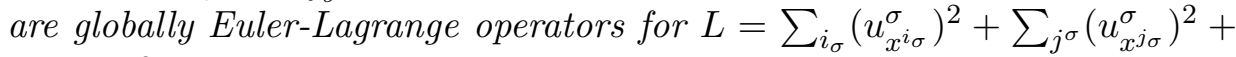
$\sum_{i}\left(u_{x^{i}}^{\mu}\right)^{2}$.

\section{References}

[1] I. M. Anderson. Natural variational principles on Riemannian manifolds Ann. of Math., Volume 120, pp 329-370, 1984.

[2] I. M. Anderson. Introduction to the variational bicomplex Contemporary Mathematics, Volume 132, pp 51-73, 1992.

[3] I. M. Anderson. On the existence of global variational principles Amer. J. Math., Volume 102, pp 781-868, 1980.

[4] I. M. Anderson and G. Thompson. The inverse problem of the calculus of variations for ordinary differential equations Memoirs Amer. Math. Soc., Volume 98, pp vi+110, 1992 .

[5] I. M. Anderson. The Variational Bicomplex http : //bib.tiera.ru/b/ 37499, (book draft, free web version, Utah, 2004), pdf.

[6] R. Bryant, S. S. Chern, R. Gardner and P. Griffiths. Essays on exterior differential systems Springer-Verlag, New York, 1990.

[7] C. Caratheodory. Variationsrechnung bei mehrfachen Integralen Acta Szeged 4, 1929.

[8] E. Cartan. Les systémes differentielles exterieurs et leurs applicationsgéométriques Herman, Paris, 1945.

[9] P. Dedecker. Calcul des variations, formes differentielles et champs geodé siques Colloques. Internat. du C.N.R.S., Strasbourg, 1953.

[10] P. Dedecker. Calcul des variations et topologie algebraique Mem. Soc. Roy. Sc. Liége, 4-e série, XIX, fase I, 1957.

[11] P. Dedecker. On the generalization of sympletic geometry to muliple integrals in the calculus of variations Lecture Notes in Math. Springer, Vol. 570, Berlin and New York, 1977.

[12] Th. De Donder. Téorie invariantive du calcul de variations Gautier-Villars, Paris, 1935. 
[13] D. G. B. Edelen. The invariance group for Hamiltonian systems of partial differential equationss Arch.Rational Mech. Anal., Volume 5 pp 95-176, 1961.

[14] D. G. B. Edelen. Nonlocal variations and local invariance of fields American Elsvier, New York, 1969.

[15] H. I. Eliasson Variational integrals in fiber bundles Proc. Sympos. Pure Math. Amer. Math. Soc., Volume 16, Providence, RI, pp 67-89 1970.

[16] P. L. Garcia, The Poincaré-Cartan invariant in the calculus of variations Sympos. Math., Volume 14, pp 219-227, 1974.

[17] P. L. Garcia, Gauge algebras, curvature and symplectic structure J. Differential Geometry, Volume 12, pp 209-246, 1977.

[18] P. L. Garcia, Critical principal connections and gauge invariance Rep. Math. Phys,Volume 13, pp 337-344, 1978.

[19] P. L. Garcia, Tangent structure of Yang-Mills equations and Hodge Theory Lecture Notes in Math. Springer, Volume 775, Berlin and New York, 1980.

[20] P. L. Garcia, A. Pérez-Rendón. Symplectic approach to the theory of quantized fields I Comm. Math Phys., Volume 13, pp 24-44, 1969.

[21] P. L. Garcia, A. Pérez-Rendón. Symplectic approch to the theory of quantized fields. II Arch. Rational Mech. Anal, Volume 43, pp 101-124, 1971.

[22] P. L. Garcia, A. Pérez-Rendón. Reducibility of the symplectic structure of minimal interactions Lecture Notes in Math. Springer, Volume 676, Berlin and New York, pp 409-433, 1978.

[23] H. Goldschmidt, S. Sternberg, The Hamilton-Cartan formalism in the calculus of variations Ann. Inst. Fourier, Volume 23, Grenoble, pp 203-267, 1973.

[24] P. Griffiths. Exterior differential systems and the calculus of variations Birkäuser, Boston, Basel, Stuttgart, 1983

[25] C. Günther,. The plysymplectic Hamiltonian formalism in the field theory and calculus of variations I: The local case J. Differential Geometry, Volume 25, pp 23-53, 1987,

[26] P. G. Henriques. Calculus of variations in the context of exterior differential systems Differential Geometry and its Applications (North-Holland), Volume 3, pp 331-372, 1993.

[27] P. G. Henriques. Well-posed variational problem with mixed endpoint conditions Differential Geometry and its Applications (North-Holland), Volume 3, pp 373393, 1993.

[28] P. G. Henriques. The Noether theorem and the reduction procedure for the variational calculus in the context of differential systems C. R. Acad. Sci. Paris Sér. I Math., Volume 317, pp 987-992, 1993.

[29] P. G. Henriques. The inverse problem of variational calculus and the problem of mixed endpoint conditions São Paulo Journal of Mathematical Science, Volume 2, pp 239-262, 2008.

[30] P. G. Henriques. The inverse problem of Variational Calculus São Paulo Journal of Mathematical Science, Volume 5, pp 233-248, 2011.

[31] R. Hermann. Differential geometry and the calculus of varitions Academic Press, New York, 1968.

[32] J. Kijowski, W. M. Tulczyjew. A symplectic framework for field theories Lecture Notes in Math. Springer, Volume 107, Berlin and New York, 1979.

[33] D. Krupka. Lagrange theory in fibered manifolds Rep. Math. Phys, Volume 2, pp 121-133, 1970.

[34] D. Krupka. A geometric theory of ordinary first order variational problems in fibered manifolds I: Critical sections J. Math Anal. Appl., Volume 49, pp 180206, 1975 . 
[35] D. Krupka. A geometric theory of ordinary first order variational problems in fibered manifolds II: Invariance J. Math Anal. Appl., Volume 49, pp 469-476, 1975.

[36] T. Lepage. Sur les champs geodésiques des integrals multiples Bull. Acad. Roy. Belg, CI. Sc., 5.éme série 22, 1936.

[37] T. Lepage. Sur les champs geodésiques des integrals multiples Bull. Acad. Roy. Belg, CI. Sc., 5.éme série 27, 1941.

[38] T. Lepage. Champs stationnaires, champs geodésiques et formes integrables I, II Bull. Acad. Roy. Belg, CI. Sc., 5.éme série 28, 1942.

[39] A. Liesen. Feldtheorie in der Variatonrechnung mehfacher Integrale I Math Annalen, Volume 171, pp 194-218 1967.

[40] A. Liesen. Feldtheorie in der Variatonrechnung mehfacher Integrale II Math Annalen, Volume 171, pp 273-292 1967.

[41] Waldyr M. Oliva and Gláucio Terra. An Inverse Problem on Vakonomic Mechanics to appear in São Paulo Journal of Mathematical Science

[42] P. J. Olver. Euler operators and conservation laws of BBM equation Math. Proc. Cam. Phil. Soc., Volume 85, pp 143-160, 1979.

[43] P. J. Olver. Applications of Lie groups to differential equations Springer, New York, 1986.

[44] R. Ouzilou. Expression symplectic des problems variationnels Sympos. Math, Volume 14, pp 85-98, 1972.

[45] H. Rund. The Hamilton-Jacobi theory in the calculus of variations Van Nostrand, Princeton, NJ, 1966.

[46] F. Takens. Symmetries, conservation laws and variational principles Lectures Notes in Mathematics, Springer, Volume 597, pp 581-603, New York, 1977.

[47] F. Takens. A global version of the inverse problem to the calculus of variations J. Differential Geometry, Volume 14, pp 543-562, 1979.

[48] W. M. Tulczyjew. The Euler-Lagrange resolution Lectures Notes in Mathematics, Springer, Volume 836, pp 22-48 S, New York, 1980.

[49] W. M. Tulczyjew. Cohomology of the Lagrange complex Ann. Scuola. Norm. Sup. Pisa, pp 217-227, 1988.

[50] M. M. Vainberg Variational Methods for tht study of Nonlinear Operators Holden-Day, S. Francisco, 1964.

[51] A. M. Vinagradov. The C-spectral sequence, Lagrangian formalism and conservation laws I, II J. Math Anal. Appl, Volume 100, pp 1-129, 1984.

[52] A. M. Vinagradov. Symmetries and conservation laws of partial differential equations: basic notions and results Acta Appl. Math., Volume 15, pp 3-22, 1989.

[53] A. M. Vinagradov. Scalar differential invariants, diffiieties and characteristic classes Mechanics, Analysis and Geometry: 200 Years after Lagrange, M. Francavglia(ed), Elsevier, Amsterdam, pp 379-416, 1991.

[54] V. Volterra. Leçons sur les Fonctions de Lignes Gautherier-Villars, Paris, 1913.

[55] H. Weyl. Geodesic fields in the calculus of variations for multiple integrals Annals of Math., Volume 36, pp 607-629 1935. 